\title{
NEUROCISTICERCOSE
}

\section{ESTUDO CLÍNICO E PATOLÓGICO DE 27 CASOS DE NECRÓPSIA}

\author{
MÁRIO RODRIGUES MONTEMÓR NETTO **, EMERSON LEANDRO GASPARETTO***, \\ LEONARDO NERCOLINI FAORO***, JORGE SÉRGIO REIS FILHO** \\ GUILHERME SANDRINI DE TONI****, ARNOLFO DE CARVALHO NETO*****, \\ LUIZ FERNANDO BLEGGI TORRES*
}

\begin{abstract}
RESUMO - Neurocisticercose é a mais frequente e disseminada neuroparasitose humana. O desenvolvimento de lesões no cérebro e leptomeninges, com consequente aparecimento de sintomas, está relacionado com a resposta imune do hospedeiro, ao número e à fase de involução dos parasitas . Relatamos os achados anatomopatológicos durante a necrópsia em 27 casos de neurocisticercose, que corresponderam a 3,1\% dos casos necropsiados no serviço. $77 \%$ dos pacientes eram masculinos, com idade variando entre 18 e 85 anos. Em 26\% dos casos havia história de alcoolismo. A análise clinico-patológica mostrou que 50\% dos casos eram da forma assintomática, $11 \%$ da forma epiléptica, $11 \%$ da forma intraventricular e $11 \%$ da forma combinada. $33 \%$ dos pacientes apresentaram crise epiléptica como fator agravante do quadro clínico. O cisticerco era único em $60 \%$ dos casos, a forma cellulosae estava presente em $82 \%$ e a racemosa em $7 \%$ dos casos, sendo os $11 \%$ restantes portadores da forma combinada. Em $30 \%$ dos pacientes a causa mortis estava relacionada com a presença do cisticerco no sistema nervoso central. Nossos achados confirmam a alta morbidade desta parasitose.
\end{abstract}

PALAVRAS-CHAVE: neurocisticercose, parasitoses, necrópsia, epilepsia, hipertensão intracraniana.

\section{Neurocysticercosis: a clinical and pathological study of 27 necropsied cases}

ABSTRACT - Neurocysticercosis is the most frequent and widespread neuroparasitosis of the human being. The development of brain and leptomeningeal lesions, with subsequent symptoms, are mainly related with the immune status of the host, and to the number and evolutional phase of the parasites. We present the pathological findings in 27 necropsies of patients with neurocysticercosis, which accounted for $3.1 \%$ of the necropsies. $77 \%$ of the patients were male and the age ranged from 18 to 85 years. In $26 \%$ there was previous history of alcoholism. Clinicopathological study showed that $50 \%$ of the cases were classified as asymptomatic form, $11 \%$ epileptic form, $11 \%$ intraventricular form and $11 \%$ combined form. $33 \%$ of the patients presented seizures as a factor of aggravation of the clinical picture. There was a single cysticercus in $60 \%$ of the cases, the cellulosae form present in $82 \%$ and the racemous form in $7 \%$ of the cases; the remaining $11 \%$ had both forms present. In $30 \%$ of the patients the cause of death was directly related with the presence of the cysticercus in the central nervous system. Our findings confirm the high morbidity of this disease.

KEY WORDS: neurocysticercosis, parasitosis, autopsy, epilepsy, intracranial hypertension.

A neurocisticercose (NCC) é a mais frequente neuroparasitose humana e uma das desordens neurológicas mais polimorfas já descritas. Suas manifestações clínicas são inespecíficas e dependem

Estudo realizado na Seção de Microscopia Eletrônica e Neuropatologia do Serviço de Anatomia Patológica, Hospital de Clínicas (HC) da Universidade Federal do Paraná (UFPR) Curitiba PR: *Professor Titular do Departamento de Patologia Médica, HC, UFPR; **Médico Residente do Serviço de Anatomia Patológica, HC, UFPR; ***Bolsista em Iniciação Científica PIBIC-CNPq da Seção de Microscopia Eletrônica e Neuropatologia, HC, UFPR. ****Médico Residente do Serviço de Radiologia Médica, HC, UFPR; ****Professor Assistente do Serviço de Radiologia Médica, HC, UFPR. Aceite: 27-junho-2000. 
do número e características das lesões, da fase de desenvolvimento e involução do parasita e da intensidade da resposta imune-inflamatória do hospedeiro ${ }^{1}$. Embora raramente constitua-se em causa mortis de pacientes adultos autopsiados, a NCC tem um impacto sócio-econômico significante, não só devido à inaptidão temporária ou permanente que ocasiona em indivíduos em idade produtiva, mas também pelo alto custo de seu diagnóstico e tratamento.

Apresentamos achados clínicos e anatomopatológicos de 27 casos de NCC em pacientes autopsiados no Serviço de Anatomia Patológica (SAP) do Hospital de Clínicas (HC) da Universidade Federal do Paraná (UFPR).

\section{MÉTODO}

Este estudo deriva de uma linha de pesquisa denominada "Neuropatologia em Necrópsia de Adultos", que tem como objetivo tabular todos os dados referentes às doenças que acometem o sistema nervoso central (SNC) de pacientes submetidos a necrópsia no HC-UFPR. A pesquisa tem o intuito de analisar epidemiologicamente as alterações neuropatológicas e fornecer um banco de dados para pesquisa e aprimoramento em neurologia, neurocirurgia, psiquiatria, psicologia e neurorradiologia.

Foram selecionados os casos de NCC entre 1987 e 1997 e estudados quanto a suas alterações clínicas e patológicas, através da revisão dos laudos de necrópsia e dos prontuários médicos. Todos os encéfalos estudados foram fixados em formol a 30\% durante o período mínimo de 3 semanas; avaliação necroscópica em cortes coronais consecutivos com guias de $1 \mathrm{~cm}$ e posteriormente fragmentos dos lobos frontal, parietal, temporal, occipital e ínsula; núcleos da base, tálamo, mesencéfalo, ponte e bulbo; verme e hemisfério cerebelar além de áreas lesadas foram amostradas para estudo histológico por técnicas de rotina (hematoxilina-eosina). Colorações especiais (luxol fast blue/Nissl e impregnação argêntica por Glees), imuno-histoquímica e análise ultraestrutural foram utilizadas quando necessárias.

Para análise estatística, compararam-se as médias de idade nos dois gêneros, utilizando-se para tal fim o teste $t$ de Student para amostras não-pareadas. Para analisar se houve diferença na frequência de NCC nos dois sexos, utilizou-se o teste chi-quadrado. Considerou-se estatisticamente significativo o valor de $p$ quando este foi igual ou inferior a 0,05 .

Todas as imagens deste estudo foram obtidas dos pacientes analisados.

\section{RESULTADOS}

No período de 1987 a 1997 foram realizadas 901 necrópsias completas no SAP- HC-UFPR em pacientes com idade acima de 15 anos. Houve 28 casos de NCC $(3,1 \%)$, sendo um dos casos excluído deste estudo por falta de dados clínicos. Todos os casos foram notificados à Secretaria de Vigilância Sanitária deste Estado, conforme determina a legislação vigente. A idade variou de 18 a 85 anos sendo que $51 \%$ dos casos encontravam-se na faixa etária entre 31 e 60 anos. Quanto ao gênero, 77,8\% eram masculinos, havendo diferença estatisticamente significativa $(\mathrm{p}<0,0001)$. A média de idade no sexo feminino foi 39,1 e no masculino 50,9 anos, não havendo diferença significativa entre os dois grupos $(\mathrm{p}=0,219)$.

Em 30\% dos casos o paciente era nascido em outro estado (5 pacientes de Minas Gerais e 3 procedentes da Bahia) e em $26 \%$ dos casos havia história de alcoolismo. Nove (33\%) pacientes apresentaram crise epiléptica como fator agravante do quadro clínico, precedendo o óbito do paciente. Destes 9 pacientes, 6 (33\%) nunca haviam manifestado previamente crise epiléptica ou outros sintomas decorrentes de lesões intracranianas. Em nossos casos, os níveis de proteínas no líquido cefaloraquidiano (LCR) estavam elevados e havia aumento na celularidade, com neutrofilia, linfocitose e eosinofilia; não foram realizados estudos imunológicos no LCR destes pacientes. Em $30 \%$ dos casos a causa mortis estava relacionada com a presença do cisticerco no SNC.

Em relação à classificação clinico-patológica, 50\% dos casos eram assintomáticos, $11 \%$ apresentavam forma epiléptica, $11 \%$ forma combinada, $11 \%$ forma intraventricular. A forma disseminada apresentou-se em 2 casos, havendo ainda um caso com apresentação meningítica, um com cerebrovascular e um com demencial. As formas espinhal ou encefalítica não foram encontradas em nosso estudo. A hipertensão intracraniana estava presente em quatro casos, associada à forma 
Tabela 1. Número e tipo de cisticerco nos 27 casos autopsiados no SAP-HC-UFPR no período de 1987 a 1997.

\begin{tabular}{ccc}
\hline Tipo do cisticerco & Número de cisticercos & Número de casos \\
\hline cellulosae & único & $15(57 \%)$ \\
cellulosae & múltiplo & $6(23 \%)$ \\
Forma racemosa e cellulosae & múltiplo & $3(12 \%)$ \\
racemosa & múltiplo & $2(8 \%)$
\end{tabular}

( 1 caso não disponível por extravio do prontuário médico)

intraventricular, meningítica ou combinada, sendo um fator de agravamento do prognóstico destes pacientes. Três casos foram submetidos a derivação ventrículo-peritoneal, sendo o diagnóstico de NCC confirmado apenas em 2 pacientes $(7,41 \%)$.

A análise neuropatológica mostrou que em $60 \%$ dos casos o cisticerco era único. A forma $C$. cellulosae estava presente em $82 \%$ dos casos, a forma racemosa $7 \%$ e em $11 \%$ dos casos encontraramse as duas formas (Tabela 1). O estágio evolutivo foi vesicular em 14\% dos casos, necrótico (colóide granular) em $36 \%$ e nódulo fibrocalcificado em $50 \%$. Outras alterações macroscópicas identificadas foram sinais de hipertensão intracraniana com dilatação ventricular em 6 casos, 7 casos com sinais de leptomeningite crônica e 1 caso de meningite basal aguda. Hidrocefalia, edema, infartos antigos e hemorragia intraparenquimatosa também foram observados. Exemplos das principais alterações detectadas estão demonstrados na Figura 1.

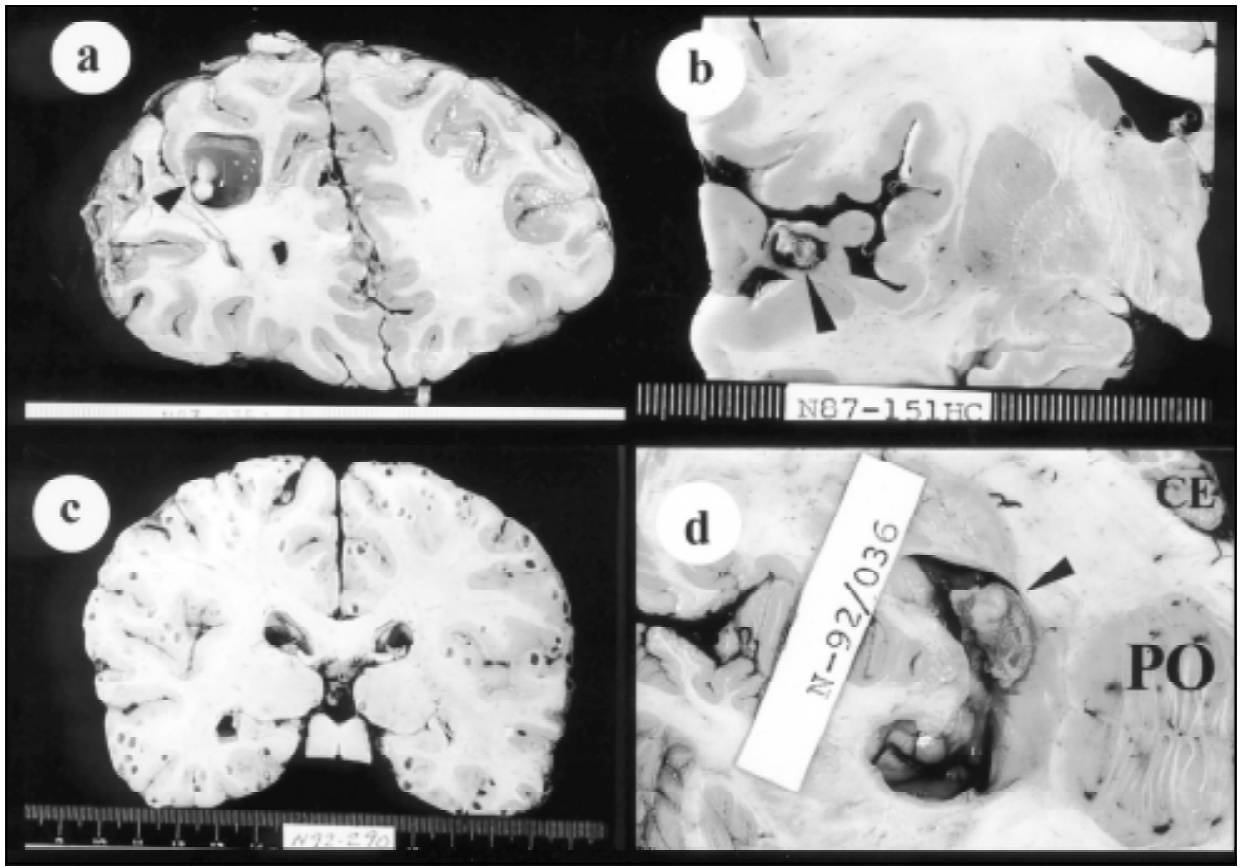

Fig 1. (a) Corte coronal de encéfalo, demonstrando cisticerco celulosae na primeira fase (cística / vesicular) viável, em região frontal. Escólex (seta) visível. (b) Corte coronal de encéfalo a nível de núcleos da base, demonstrando em fissura Sylviana presença de cisticerco na segunda fase (necrótica) com halo inflamatório hiperêmico circunjacente (seta). (c) Corte coronal de encéfalo a nível de porção posterior do tálamo, demonstrando formas disseminadas contendo dezenas de parasitas intraparenquimatosos. (d) Detalhe de corte do tronco cerebral ao nível de quarto ventrículo demonstrando forma intraventricular com cisticerco na primeira fase de evolução (vesicular), viável, com escólex (seta) evidenciável ( $P O$, ponte; $C E$, cerebelo). 
Separando-se o grupo em que o cisticerco era único (16 casos), foram obervados 6 casos onde este localizava-se no hemisfério cerebral esquerdo e 5 casos no direito, havendo ainda 3 casos com cisticerco intraventricular e 2 casos em núcleos da base.

\section{DISCUSSÃO}

NCC é doença endêmica na maioria dos países em desenvolvimento na América Latina e Central, África do Sul, Ásia (China, Índia, e Indonésia), países da Europa Oriental (Polônia e Romênia), bem como em Portugal e Espanha e entre grupos sociais imigrantes para países desenvolvidos ${ }^{2-6}$.

A frequência de NCC varia de 0,1 a 3,6\% nas séries de pacientes autopsiados em hospitais gerais em vários países de América Latina, África e Ásia ${ }^{7-9}$. Embora os estudos baseados em autópsias constituam um método útil para calcular a prevalência de NCC, eles apresentam limitações. Poucos profissionais com habilidades adequadas em neuropatologia estão disponíveis em áreas onde NCC é endêmica, e muitos dos estudos existentes baseados em achados de autópsia foram realizados por patologistas gerais sem menção a metodologia usada para estudo do encéfalo. Exemplo de tal situação pode ser obtido dos registros do SAP do Hospital de Clínicas da UFPR, onde a frequência da NCC no período de 1977 a 1986 era de $0,53 \%$, sendo que a partir de 1987 a análise do encéfalo passou a ser feita por um neuropatologista, e subsequentemente no período de 1987 a 1997 a frequência de NCC elevou-se para 3,1\%. Embora outros fatores pudessem explicar a diferença em prevalência de NCC entre os dois períodos indicados acima, o mais importante foi certamente a mudança no método usado para exame do encéfalo. Assim a prevalência de NCC foi menosprezada em algumas das séries baseadas no estudo de autópsias, fato também observado por Pittella ${ }^{10}$.

NCC afeta com maior frequência adultos do sexo masculino, sendo assintomática na maioria dos $\operatorname{casos}^{1,7}$. Em nosso estudo $77 \%$ dos casos eram do sexo masculino e $50 \%$ dos casos eram assintomáticos. Entretanto, a NCC pode causar uma variedade de manifestações clínicas, sendo causa possível de dificuldade de diagnóstico ${ }^{10}$. Este polimorfismo clínico da NCC é determinado por: 1) número de lesões (cisticerco único ou múltiplo); 2) localização das lesões no SNC (subaracnóide, intracerebral, intraventricular, intramedular); 3) tipo de cisticerco (cisticerco cellulosae ou racemosus); 4) fase de desenvolvimento e involução do parasita (vesicular ou viável, necrótico, nódulo fibrocalcificado); e 5) intensidade da resposta imune-inflamatória do hospedeiro (nenhuma reação inflamatória, leptomeningite, encefalite, ependimite granular, arterite).

O diagnóstico através da história clínica e do exame físico pode ajudar em alguns casos, entretanto o diagnóstico depende da neuroimagem e análise do $\mathrm{LCR}^{11-14}$. O diagnóstico de NCC durante o internamento foi confirmado em apenas 2 pacientes do nosso estudo, sendo os demais $(\mathrm{n}=25)$ diagnosticados por necrópsia. Em um estudo, a forma sintomática foi evidenciada em $60 \%$ dos casos, sendo confirmada por tomografica computadorizada (TAC) ${ }^{15}$; entretanto, neste estudo, a investigação nem sempre foi feita através de estudos radiográficos sensíveis, desta forma o diagnóstico em vida foi estabelecido em apenas 2 casos. Convém ressaltar que vários de nossos casos haviam internado por outra patologia e o diagnóstico de NCC foi achado ocasional de necrópsia.

Os mecanismos pelos quais as lesões do cisticerco se desenvolvem no cérebro, medula espinhal e leptomeninges dependem de uma combinação de fatores do hospeiro como a resposta imuneinflamatória, presença de alterações compressivas e processos obstrutivos induzidos pelo parasita ${ }^{10,16}$. Alterações compressivas podem ser resultantes do efeito de massa produzido por um cisticerco volumoso ou de um número grande de cisticercos pequenos. Processos obstrutivos podem surgir do bloqueio da circulação do LCR por cisticercos localizados nos ventrículos ou no espaço subaracnoideo; porém, com maior frequência, a obstrução é induzida pelo desenvolvimento de ependimite granular nos casos de NCC ventricular, e leptomeningite crônica fibrosante nos casos de NCC subaracnóidea. A análise neuropatológica dos casos do nosso estudo evidenciou sinais de hipertensão intracraniana com dilatação ventricular em 6 casos, 7 casos com sinais de leptomeningite crônica e 1 caso de meningite basal aguda. Outras alterações encontradas foram hidrocefalia, edema, infartos antigos e hemorragia intraparenquimatosa. 
Os cisticercos podem permanecer viáveis no SNC por 1 a 3 anos, dependendo da tolerância imune do hospedeiro ${ }^{1,10}$. Morfologicamente, três fases do desenvolvimento e da regressão do cisticerco no SNC são reconhecidas. O número de parasitas é variável na NCC ${ }^{8,10}$. Em 20 a 53\% dos casos são achados cisticerco solitários, sendo que em nosso estudo $60 \%$ dos casos apresentava cisticerco único. Quando múltiplos, os cisticercos normalmente estão em um número pequeno. $\mathrm{O}$ achado de centenas de parasitas caracteriza a forma disseminada, a qual é rara. Em nossa casúística foram observados casos de formas com Cysticercus cellulosae e Cysticercus racemosus; a coexistência entre as duas formas é observada em $10 \%$ dos $\operatorname{casos}^{10}$, sendo que em nossa casuística havia coexistência em $11 \%$ dos casos.

Evolutivamente, Pittella ${ }^{10}$ realizou um estudo importante, em que correlacionou a fase evolutiva do cisticerco, suas características, e aspectos radiológicos. A primeira fase, também definida como cística ou vesicular, consiste de cisticerco viável e composto de uma membrana bem definida com líquido no seu interior e contém apenas um escolex; observa-se discreto a moderado inflitrado inflamatório linfoplasmocitário e alguns eosinófilos que podem ou não estar presentes ao redor do cisticerco. Quando o parasita se localiza em tecido nervoso, pode ser vista discreta astrocitose fibrilar mais externamente. Radiologicamente, aparece como uma imagem hipodensa e sem contraste ao redor da lesão, solitário ou múltiplo, com 5-10 mm em diâmetro, contendo um escolex. A segunda fase (fase necrótica, também conhecida como colóide ou granular), caracteriza-se por necrose do parasita associado a processo inflamatório, aparecendo como uma estrutura eosinofílica na qual componentes do cisto e escolex se apresentam em várias fases de desintegração. Existe uma reação tipo corpo estranho com células gigantes multinucleadas, macrófagos e número pequeno de neutrófilos; também são observados cristais de colesterol. Neoformação de tecido conjuntivo denso e fibroso infiltrado ou circundado por células linfoplasmocitárias e alguns eosinófilos ao redor do cisticerco necrótico podem ser vistos. Necrose e/ou edema do tecido nervoso circunvizinho pode estar presente em alguns casos, particularmente em pacientes tratados com praziquantel e que morreram logo após, como também em pacientes com uma forte resposta imune-inflamatória (encefalite cisticercótica). Com o tempo, a fibrose se desenvolve ocupando progressivamente a lesão. A terceira fase (fase final, nódulo fibrocalcificado) apresenta cisticerco cellulosae como nódulo fibrocalcificado, sendo que a fibrose ocupa a lesão inteira, associado ou não com inflitrado linfoplasmocitário residual. Macroscopicamente, o nódulo fibrocalcificado residual tem consistência dura e é menor que o cisto observado na fase anterior. Em aproximadamente 10\% dos casos de NCC, só pode ser identificado através de exame microscópico. Radiologicamente, observa-se nódulo calcificado, sendo sua densidade maior a observada através do contraste. Ja quando a $3^{\text {a }}$ fase relaciona-se a cisticerco racemoso, este induz uma reação inflamatória e uma fibrose mais intensa, o

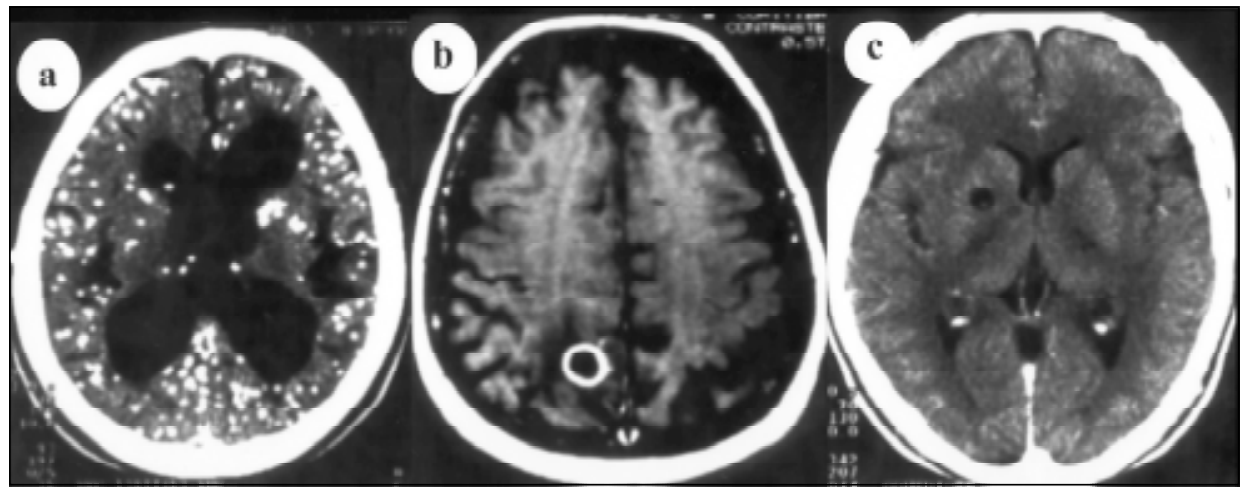

Fig 2. (a) Estágio fibro-calcificado. Imagem de TAC demonstrando múltiplas calcificações no parênquima cerebral. (b) Imagem de RNM (ponderada em T1/pós-contraste) demonstrando cisto em estágio necrótico ou granulomatoso, com sinais de quebra de barreira hematoencefálica e involução do cisto. Área de impregnação anelar de contraste. (c) Estágio vesicular. Imagem de TAC demonstrando cisto isolado na profundidade do hemisfério cerebral direito. 
que sugere formação ativa e contínua novos antígenos, sem o desenvolvimento de tolerância imune, comumente observado com cisticerco cellulosae. A TAC pode permitir sua melhor visualização quando a imagem observada é um agrupamento de cistos multiloculados organizado como um "cacho de uvas".

A TAC e a ressonância nuclear magnética (RNM) melhoraram sobremaneira a precisão do diagnóstico de $\mathrm{NCC}^{14,17-21}$. Os achados neurorradiológicos dependem do tipo de cisticerco, da fase de desenvolvimento e involução da larva e da localização e número de cisto (Fig 2). A injeção de contraste é de grande valor no diagnóstico de NCC. A reação inflamatória ao redor do cisticerco necrótico causa ruptura da barreira de hemato-encefálica e permite que o contraste penetre ao redor da lesão. TAC permite identificar outras lesões associadas com NCC, como leptomeningite, hidrocefalia, infarto e edema cerebral. A RMN é superior à TAC na demonstração do cisticerco viável, e também é mais apropriada para comprovar o escólex dentro do cisto. Nossos dois pacientes com diagnóstico em vida apresentavam, aos cortes tomográficos, uma lesão circular e hipodensa cercada por edema que se acentuava com o contraste, caracterizando a fase necrótica do cisticerco (Fig 2).

Análise do LCR, junto com os dados clínicos e achados de neuroimagem, é essencial para o diagnóstico de $\mathrm{NCC}^{11,13,18,22}$. O aparecimento de células inflamatórias e anticorpos específicos no LCR coincide com a degeneração do cisticerco e consequente intensificação da resposta imuneinflamatória do hospedeiro, em particular quando o parasita se localiza no espaço subaracnóideo e produz leptomeningite. Reação de fixação de complemento, imunofluorescência indireta, hemoaglutinação passiva e análise imunoenzimática constituem métodos diagnósticos complementares com alta sensibilidade e especificidade ${ }^{23,24}$.

O tratamento da NCC ainda é objeto de debate; algumas drogas (como o prazinquantel) podem induzir regressão dos cistos; outras vezes a derivação ventrículo-peritoneal é utilizada como paliativa à meningite obstrutiva ${ }^{18,25-30}$. Nossos dois pacientes com diagnóstico em vida de NCC foram tratados com prazinquantel, mas não obtiveram sucesso na terapêutica. Em qualquer das situações a NCC apresenta alta morbidade sendo responsável por profundo desgaste sócio-econômico em países endêmicos.

\section{REFERÊNCIAS}

1. Shandera WX, White AC Jr, Chen JC, Diaz P, Armstrong R. Neurocysticercosis in Houston, Texas: a report of 112 cases. Medicine (Baltimore) 1994;73:37-52.

2. Villa AM, Monteverde DA, Rodriguez W, Boero A, Sica RE. Neurocysticercosis in a hospital of the city of Buenos Aires: study of 11 cases. Arq Neuropsiquiatr 1993;51:333-336.

3. Garcia HH, Gilman RH, Tovar MA, et al. Factors associated with Taenia solium cysticercosis: analysis of nine hundred forty-six Peruvian neurologic patients. Cysticercosis Working Group in Peru. Am J Trop Med Hyg, 1995;52:145-148.

4. Albuquerque ES, Galhardo I. Neurocysticercosis in the State of Rio Grande do Norte: report of 8 cases. Arq Neuropsiquiatr 1995;53:464-470.

5. Takayanagui OM, Castro e Silva AA, Santiago RC, Odashima NS, Terra VC, Takayanagui AM. Compulsory notification of cysticercosis in Ribeiräo Preto-SP, Brazil. Arq Neuropsiquiatr 1996;54:557-564.

6. Gonçalves-Coelho T, Coelho MDG. Neurocysticercosis in Paraiba, Northeast Brazil: an endemic area? Arq Neuropsiquiatr 1996;54:565-570.

7. Veliath AJ, Ratnakar C, Thakur LC. Cysticercosis in South India. J Trop Med Hyg 1985;88:25-29.

8. Costa-Cruz, JM, Rocha A, Silva AM, et al. Ocorrência de neurocisticercose em necrópsias realizadas em Uberlândia, Minas Gerais, Brasil. Arq Neuropsiquiatr 1995;53:227-232.

9. Chimelli L, Lovalho AF, Takayanagui OM. Neurocysticercosis: contribution of autopsies in the consolidation of mandatory notification in Ribeiräo Preto-SP, Brazil. Arq Neuropsiquiatr 1998;56:577-584.

10. Pittella JE. Neurocysticercosis. Brain Pathol 1997;7:681-693.

11. St Geme JW, Maldonado YA, Enzmann D, Hotez PJ, Overturf GD, Schantz PM. Diagnosis and management of neurocysticercosis in children. Pediatr Infect Dis J 1993;12:455-461.

12. Ramos Kuri M, Montoya RM, Padilla A, et al. Immunodiagnosis of neurocysticercosis: disappointing performance of serology (enzyme-linked immunosorbent assay) in an unbiased sample of neurological patients. Arch Neurol 1992;49:633-636.

13. Machado LR. The cerebrospinal fluid and neurocysticercosis: evolutional aspects of the cellular inflammatory response. Arq Neuropsiquiatr 1987;45:353-363.

14. Machado LR, Nobrega JP, Barros NG, Livramento JA, Bacheschi LA, Spina França A. Computed tomography in neurocysticercosis: a 10-year long evolution analysis of 100 patients with an appraisal of a new classification. Arq Neuropsiquiatr 1990;48:414-418. 
15. Monteiro L, Coelho T, Stocker A. Neurocysticercosis: a review of 231 cases. Infection 1992;20:61-65.

16. Ostrosky Zeichner L, Estañol B. Immunopathogenesis of neurocysticercosis: is damage mediated by the host immune response? Int J Parasitol 1999;29:649-650.

17. Bittencourt PR, Costa AJ, Oliveira TV, Gracia CM, Gorz AM, Mazer S. Clinical, radiological and cerebrospinal fluid presentation of neurocysticercosis: a prospective study. Arq Neuropsiquiatr 1990;48:286-295.

18. Rajshekhar V, Chacko G, Haran RP, Chandy MJ, Chandi SM. Clinicoradiological and pathological correlations in patients with solitary cysticercus granuloma and epilepsy: focus on presence of the parasite and oedema formation. J Neurol Neurosurg Psychiatry 1995;59:284-286.

19. Galhardo I, Coutinho MO, De Albuquerque ES, Medeiros LO, Dantas JO. Neurocysticercosis in Rio Grande do Norte before and after computed tomography: report of a case. Arq Neuropsiquiatr 1993;51:541-545.

20. Ciftçi E, Diaz Marchan PJ, Hayman LA. Intradural-extramedullary spinal cysticercosis: MR imaging findings. Comput Med Imaging Graph 1999;23:161-164.

21. Sheth TN, Pilon L, Keystone J, Kucharczyk W. Persistent MR contrast enhancement of calcified neurocysticercosis lesions. Am J Neuroradiol 1998;19:79-82.

22. Bueno EC, Vaz AJ, Oliveira CA, et al. Analysis of cells in cerebrospinal fluid from patients with neurocysticercosis by means of flow cytometry. Cytometry 1999;38:106-110.

23. Correa D, Sarti E, Tapia Romero R, et al. Antigens and antibodies in sera from human cases of epilepsy or taeniasis from an area of Mexico where Taenia solium cysticercosis is endemic. Ann Trop Med Parasitol 1999;93: 69-74.

24. Dekumyoy P, Vanijanonta S, Waikagul J, Sa-nguankiat S, Danis M. Use of delipidized antigens of Taenia solium metacestodes in IgG-ELISA for detection of neurocysticercosis. Southeast Asian J Trop Med Public Health 1998;29:572-578.

25. Colli BO, Martelli N, Assirati JA Júnior, et al. Cysticercosis of the central nervous system: I. Surgical treatment of cerebral cysticercosis: a 23 years experience in the Hospital das Clínicas of Ribeirão Preto Medical School. Arq Neuropsiquiatr 1994;52:166-186.

26. Kramer LDM. Medical treatment of cysticercosis-ineffective. Arch Neurol 1995;52:101-102

27. Agapejev S, Silva MD, Ueda AK. Severe forms of neurocysticercosis: treatment with albendazole. Arq Neuropsiquiatr 1996;54:82-93.

28. Baranwal AK, Singhi PD, Khandelwal N, Singhi SC. Albendazole therapy in children with focal seizures and single small enhancing computerized tomographic lesions: a randomized, placebo-controlled, double blind trial. Pediatr Infect Dis J 1998;17:696-700.

29. Marques MP, Takayanagui OM, Bonato PS, Santos SR, Lanchote VL. Enantioselective kinetic disposition of albendazole sulfoxide in patients with neurocysticercosis. Chirality 1999;11:218-223.

30. Jung H, Medina L, García L, Fuentes I, Moreno Esparza R. Absorption studies of albendazole and some physicochemical properties of the drug and its metabolite albendazole sulphoxide. J Pharm Pharmacol 1998;50:43-48. 